\title{
Impacts of Uterine Leiomyomata on Women's Quality of Life
}

\author{
Marwa Mohamed Awad Allah ${ }^{1}$, Faisal Mohammed Salih Basama ${ }^{2}$, Amir Elnahas ${ }^{3 *}$ and Ameer Osman Abaker \\ ${ }^{1}$ Omdurman Maternity Teaching Hospital, Omdurman, Sudan \\ ${ }^{2,3}$ FRCOG, Senior Attending Physician in Obstetrics and Gynecology, Sidra Medicine, Assistant Professor of Clinical Obstetrics and Gynecology, \\ Weill Cornell Medical College-Qatar, Doha, Qatar \\ ${ }^{4}$ Consultant obstetrician and Gynecologist, Ibrahim Malik Teaching Hospital, Associate professor of Obstetrics and Gynecology, Department \\ Obstetrics and Gynecology, University of Khartoum, Khartoum, Sudan
}

Received: 制 July 11, 2018; Published: 制 August 13, 2018

*Corresponding author: Amir Elnahas, Senior Consultant obstetrician and gynecologist, Sidra medical centre, PO Box 26999, Doha, Qatar, Assistant professor of clinical obstetrics and gynecology, Weil Cornell medical college, Qatar

\section{Abstract}

Background: Most women with Uterine Leiomyomata (UL) attribute various negative experiences in their lifetime to symptoms related to UL such as: abnormal uterine bleeding, various pressure symptoms and pelvic pain. Most of the research on UL examined the efficacy of treatment modalities, but only few researchers have addressed the impact of the disease on the women's HealthRelated Quality of Life (HRQL).

Methods: This is a descriptive, prospective, hospital-based qualitative study. The data was collected by: Focus Group (FG) interviews and by structured questionnaire. 200 women with symptomatic UL were recruited over a period of 6 months. The data was analyzed using the Statistical Package for Social Sciences (SPSS) data analysis tool, Version 20.

Results: The majority of women (98\%) graded their symptoms as moderate or severe. The mostly affected group are those in the reproductive age (20 to 39 years). $69 \%$ of the employed women claimed that the disease has affected their work and $55 \%$ of them reported UL as a reason for their absence from work. $27.2 \%$ of all women think that the disease has moderately restricted their social and physical activities, whereas $66.4 \%$ think the impact on their work is severe. $28.3 \%$ of all women think that their sexual life was moderately dysfunctional because of the disease and $52.2 \%$ think the effect was severe. $36.8 \%$ and $61.6 \%$ of all women reported moderate to severe psychological concerns respectively, such as: the sensation of fear, feeling of unfairness, and discouragement.

Conclusions: Uterine leiomyomata are a common health concern for women in the child-bearing age. Symptomatic UL has apparently a negative impact on: women HRQL, sexual and social lives and it appears to affect their work performance. There is no significant correlation between the women symptoms and type of employment; however, the study is suggestive of a possible positive correlation between the women severity of symptoms and: social activities, energy, mood, sense of control, and sexual function.

\section{Introduction}

Uterine Leiomyoma (UL) is the most prevalent benigngynecological tumor in the female population. The tumor originates from the smooth muscle of the myometrium [1-3]. As many as 1 in 5 women in child-bearing age may have UL, and between 20 to $80 \%$ of all women may have the tumor by the age of 50 [4]. It seems that UL is more common in black than white Caucasian women $[5,6]$. The exact a etiology of UL is unknown, but there is considerable evidence that it might be due to a combination of factors, such as: hormonal, environmental, chromosomal, genetic drivers, cellular modulation, and phenotypical changes [7-9,2,10,11]. The UL have different sizes, locations and growth pattern and it may attain enough volume to significantly distort the uterine cavity and endometrial surface and affect the menstrual flow [12,13]. It may also affect reproductive outcomes and may be associated with pregnancy loss, preterm birth, dystocia and fetal malpresentation [14-17] 
Many women with UL may have symptoms like:

a) Heavy and/or prolonged menses

b) Dysmenorrhea

c) Pressure symptoms on the adjacent structures like the: bladder, rectum and vagina.

d) Abdominal enlargement

e) Dyspareunia

The impact and the severity of the symptoms depend on: the size, the number and the site of the tumor, and black women are more likely to have severe or very severe symptoms [18]. Huge leiomyomata may be mistaken for pregnancy and in some conservative societies this can cause significant social embarrassment, especially to the single woman leading to lower self-esteem and negative impact on the quality of life. Although UL have no apparent effect on: libido, arousal, lubrication or, orgasm [19]. however, some women with UL reported pain during sexual intercourse [20-22], and this could possibly lead to anxiety, apprehension and sexual dysfunction and understandably sexual dissatisfaction and fear of pain during sex may dissuade the woman from sexual activity and can potentially lead to marital disharmony [18]. The association between UL and subfertility is controversial and lacks convincing evidence; however, a systemic review by Pritts and coworkers (2009) showed that UL, regardless of location, were associated with: reduction in pregnancy rates, live birth rates, and increase in miscarriage. These effects vary according to the type of fibroid itself, but the effect is more prominent with sub-mucous UL perhaps by distorting the implantation site [16]. UL are the leading cause of hysterectomy in the USA [23] and hysterectomy is considered a definitive treatment; however, recurrence and loss of reproductive potential could have psychosocial impacts on these women. Nonetheless, there are several alternative medical and less radical surgical modalities for the treatment of UL and the choice of treatment is dictated by many factors such as: the patient's desire to become pregnant in the future, the importance of uterine preservation, the severity of symptoms, and the tumor characteristics.

\section{Uterine Leiomyomata and the Quality of Life}

Heavy menstrual periods, prolonged bleeding, frequent periods, inter-menstrual bleeding, irregular and unpredicted periods, abdominal discomfort, pain, pressure symptoms, anemia and fatigue are among the commonly reported symptoms [12,13]. The severity and the unpredictability of such symptoms are more than likely to affect the quality of life of women negatively, making them feel not in control, irritable and anxious and limit and interfered with physical activities and make them worry about their health, relationships, work performance and progression [24-26]. Despite that many women with UL: delayed treatment, sought more than two physicians for consultation and some waited an average of 3.6 years before seeking treatment [22]. There were few studies examining the effect of UL on women 's sexual satisfaction, and even fewer on the effects of different treatment options on sexual satisfaction [22]. The effect of the location of UL on the causation of dyspareunia is controversial; however, women with very large tumor recorded the worst sexual satisfaction and most pain $[21,19]$. The number and size of the tumor do not appear to influence the incidence or intensity of dyspareunia; however, fundal and anteriorly-placed leiomyomata seem to cause higher-intensity dyspareunia and it is more distressing if the tumor is near the cervix [19]. UL seems to interfere with sexual satisfaction probably from the dyspareunia, but the tumor seems to have no effect on libido, arousal, lubrication or orgasm [19]. The prevalence of UL in pregnancy is 3 to $10 \%$. Complication such as red degeneration, spontaneous miscarriage, preterm labor, intrauterine growth restriction, dysfunctional labor, fetal malposition, postpartum hemorrhage, retained placenta and higher Caesarean section rates have all been reported in association with UL $[26-30,14,17]$. Many women with UL, especially those with menorrhagia, felt "helpless" or "trapped" and claimed that UL have negative impact on their mental wellbeing, lifestyle and marital life $[31,32]$.

\section{The Rationale}

UL are one of the most common benign tumors during women's reproductive age and despite that there are only few studies that have adequately assessed the impact of UL on women's productivity and quality of life. This research aims to study the effect of UL on the women's quality of life and the objectives are to explore the impact of UL on the women's:

Work performance both at home and at office

a) Social relationship

b) Sexual function

c) Body image

d) Concerns regarding fertility and pregnancy complications

e) Reaction to the various treatment modalities

\section{The Methods}

\section{The Site}

Omdurman Maternity Teaching Hospital is the largest specialized maternity hospital in Sudan with average number of deliveries of more than 25000 per annum.

\section{The Study Period}

The study spanned over 7 months period; starting on the first of August 2014 and the last of day of recruitment was the 28th of February 2015. 


\section{The Study Population}

The study population was all women who were diagnosed with UL during the time of the study at Omdurman Maternity Teaching Hospital. The women were presenting with various gynecological problems and some of them were pregnant.

\section{The Study Sample Size}

The sample size was 200 and was calculated according to the equation:

$$
\begin{aligned}
& X=Z(c / 100)^{2} r(100-r) \\
& N=N X /\left((N-1) E^{2}+X\right) \\
& E=\operatorname{Sqrt}[(N-n) x / n(N-1)]
\end{aligned}
$$

Where $\mathrm{N}$ is the population size, $\mathrm{r}$ is the fraction of responses of interest, and Z(c/100) is the critical value for the confidence level c.

\section{Data Collection}

The data was collected by; detailed and structured questionnaires and by direct interview conducted by Dr Marwa Mohamed Awad Allah aided by trained colleagues.

\section{Inclusion Criteria}

The study included women attending the obstetrical or the gynecological clinics with symptoms related to UL, or diagnosed with UL, or underwent surgery for UL at Omdurman Maternity Hospital during the study period.

\section{The Exclusion Criteria}

Women who had pelvic masses proved to be not UL and women who are not willing to participate in the study were excluded.

\section{Data Analysis}

The data was analyzed using the Statistical Package for Social Sciences (SPSS) data analysis tool, Version 20.

\section{Ethical Approval}

The study was approved by the Ethics Committee of the Sudan Medical Specialization Board. Permission was also obtained locally from: the medical director of Omdurman Maternity Teaching Hospital and the local hospital ethical committee. Verbal and written consent was obtained from all the participants.

\section{The Results}

A total of 200 women diagnosed with UL attending Omdurman Maternity Teaching Hospital were recruited. 187 (93.5\%) of the women were in the age group (20-39) years and 13 (6.5\%) were 40 years of age or above (Table 1). 129 women (64.5\%) were married, $60(30 \%)$ were single and 11 (5.5\%) were divorced see Table 1. 127 of the women (63.5\%) were nulliparous and 73 (36.5\%) were parous, see Table 1. 29 (39.7\%) of the parous women are para
1, $27(37 \%)$ are para 2, $11(15.1 \%)$ are para 3 and $6(8.2 \%)$ are para 4 or more, see pie chart (1). 60 women (30\%) completed primary education, 80 (40\%) completed secondary school and $60(30 \%)$ attained higher education (Table 1). 50 of all women (25\%) achieved undergraduate qualification and $10(5 \%)$ had postgraduate qualifications, see Table 1.58 women (29\%) have jobs and $142(71 \%)$ are housewives (Table 1). 91 women were pregnant (45.5\%): 18 of them (19.8\%) had miscarriage and the pregnancies continued in $73(80.2 \%)$ (Table 1$) .48$ women out of the continued pregnancies (65.8\%) delivered vaginally, whereas $25(34.2 \%)$ had Caesarean delivery, see (Table 1). 10 women (5\%) were labelled as anemic with hemoglobin less than $10.5 \mathrm{~g} / \mathrm{dl}$ and 3 of them required blood transfusion, see Table 1. 38 of all the women (19\%) have a history of subfertility, see Table 1 . but the rate would have been higher (29.5\%), if we take into consideration that only 129 women were married. 196 women (98\%) graded their symsssptoms as moderate or severe (Table 2). Four (2\%) women described their menstrual loss and duration as mild, 80 (40\%) claimed moderately heavy and/or prolonged periods and in 116 of the women (58\%) the bleeding was described as severe and/or prolonged (Table 2). 21 women $(10.5 \%)$ thought that they were mildly affected by the pressure symptoms of the leiomyomata, the effect was moderate in 78 (39\%) and severe in 101(50.5\%), see table (2). The impact of the leiomyomata on the psychological wellbeing of the women was rated as mild by 3 women (1.5\%), moderate by 74 (37\%) and severe by 123 (61.5\%), see Table 2.13 women (6.5\%) think that the symptoms caused by the leiomyomata have mildly affected their physical activity, the effect was moderate in $54(27 \%)$ and severe in 133 (66.5\%), see Table 2. 8 women (4\%) thought that the leiomyomata had mild negative impact on their mode and energy, the impact was moderate in 49 (24.5\%) and severe in 143 (72.5\%), see table (2). 18 women (9\%) thought that the symptoms have made them loose control, in 37 women $(18.5 \%)$ the effect was moderate, and it was severe in 145 (72.5\%), see Table 2. Of the 200 women surveyed only 140 (70\%) were happy to comment on the effect of the leiomyomata on their sexual satisfaction, 27 women (19.3\%) think the leiomyomata and the related symptoms have mildly affected their sexual satisfaction, the effect was moderate in 20 women (28.6\%) and it was severe in 73 (52.1\%). 6 of the 11 (54.4\%) divorcee claimed that the UL had affected their sexual and impacted on their marital lives and resulted in disharmony. Some of the employed women claimed that UL-related symptoms have:
a) Affected their work $(40=69 \%)$
b) Put them off work $(32=55 \%)$
c) Forced them to be off work for $1-4$ days $(10=17.2 \%)$
d) Reduced their productivity $(21=36.2 \%)$
e) Lowered their work satisfaction $(20=34.5 \%)$
f) Hampered their career progression $(38=65.5 \%)$ 
Table 1: showing the women's demographic features.

\begin{tabular}{|c|c|c|c|}
\hline \multirow{2}{*}{ Age in years } & $20-39$ & \multicolumn{2}{|c|}{$\geq 40$} \\
\hline & $178(93.5 \%)$ & \multicolumn{2}{|c|}{$13(6.5 \%)$} \\
\hline \multirow{2}{*}{ Marital status } & Married & Single & Divorcee \\
\hline & $129(64.5 \%)$ & $60(30 \%)$ & $11(5.5 \%)$ \\
\hline Subfertility & $38(19 \%)$ & & \\
\hline \multirow{2}{*}{ Parity } & Nulliparous & \multicolumn{2}{|c|}{ Parous } \\
\hline & $127(63.5 \%)$ & \multicolumn{2}{|c|}{$73(36.5 \%)$} \\
\hline \multirow{2}{*}{ Literacy } & Primary education & Secondary education & High Education \\
\hline & $60(30 \%)$ & $80(40 \%)$ & $60(30 \%)$ \\
\hline \multirow{2}{*}{ Under and Postgraduate education } & Undergraduate & \multicolumn{2}{|c|}{ Postgraduate } \\
\hline & $50(25 \%)$ & \multicolumn{2}{|c|}{$10(5 \%)$} \\
\hline \multirow{2}{*}{ Employment } & Employed & \multicolumn{2}{|c|}{ Housewives } \\
\hline & $58(29 \%)$ & \multicolumn{2}{|c|}{$142(71 \%)$} \\
\hline \multirow{2}{*}{ Pregnancy } & Pregnant & \multicolumn{2}{|c|}{ Non-pregnant } \\
\hline & $91(45.5 \%)$ & \multicolumn{2}{|c|}{$109(55.5 \%)$} \\
\hline \multirow{2}{*}{ Pregnancy Outcome } & Miscarriage & \multicolumn{2}{|c|}{ Continued pregnancy } \\
\hline & $18(19.8 \%)$ & \multicolumn{2}{|c|}{$73(80.2 \%)$} \\
\hline \multirow{2}{*}{ Mode of Delivery } & Normal delivery & \multicolumn{2}{|c|}{ Caesarean section } \\
\hline & $48(65.8 \%)$ & \multicolumn{2}{|c|}{$25(34.2 \%)$} \\
\hline${ }^{*}$ Anemia (hemoglobin less than $10.5 \mathrm{~g} / \mathrm{dl}$ ) & $10(5 \%)$ & & \\
\hline
\end{tabular}

*3 patients received blood transfusion

Table 2: outlines the symptoms caused by the UL in the whole women population.

\begin{tabular}{|c|c|c|c|c|c|c|c|c|}
\hline \multirow{2}{*}{ Symptoms } & \multicolumn{2}{|c|}{ Mild } & \multicolumn{2}{|c|}{ Moderate } & \multicolumn{2}{|c|}{ Severe } & \multirow{2}{*}{ Total } & \multirow{2}{*}{ Percentage } \\
\hline & Number & Percentage & Number & Percentage & Number & Percentage & & \\
\hline $\begin{array}{l}\text { The grade of } \\
\text { symptoms }\end{array}$ & 4 & $2 \%$ & 74 & $37 \%$ & 122 & $61 \%$ & 200 & $100 \%$ \\
\hline $\begin{array}{l}\text { Menstrual loss } \\
\text { and duration }\end{array}$ & 4 & $2 \%$ & 80 & $40 \%$ & 116 & $58 \%$ & 200 & $100 \%$ \\
\hline $\begin{array}{c}\text { Pressure } \\
\text { symptoms }\end{array}$ & 21 & $10.5 \%$ & 78 & $39 \%$ & 101 & $50.5 \%$ & 200 & $100 \%$ \\
\hline $\begin{array}{l}\text { Psychological } \\
\text { impact }\end{array}$ & 3 & $1.5 \%$ & 74 & $37 \%$ & 123 & $61.5 \%$ & 200 & $100 \%$ \\
\hline $\begin{array}{c}\text { Effect on } \\
\text { physical } \\
\text { activity }\end{array}$ & 13 & $6.5 \%$ & 54 & $27 \%$ & 133 & $66.5 \%$ & 200 & $100 \%$ \\
\hline $\begin{array}{l}\text { Effect on } \\
\text { mode and } \\
\text { energy }\end{array}$ & 8 & $4.0 \%$ & 49 & $24.5 \%$ & 143 & $71.5 \%$ & 200 & $100 \%$ \\
\hline $\begin{array}{l}\text { The sense of } \\
\text { control }\end{array}$ & 18 & $9.0 \%$ & 37 & $18.5 \%$ & 145 & $72.5 \%$ & 200 & $100 \%$ \\
\hline $\begin{array}{c}\text { Sexual } \\
\text { satisfaction }\end{array}$ & 27 & $19.3 \%$ & 40 & $28.6 \%$ & 73 & $52.1 \%$ & 140 & $100 \%$ \\
\hline
\end{tabular}

Table 3 The association was strong and significant between the intensity of the symptoms and the followings:

Table 3: outlines the effect of UL-related symptoms on work.

\begin{tabular}{|c|c|c|c|c|}
\hline & $\begin{array}{c}\text { Affected number and } \\
\text { percentage }\end{array}$ & $\begin{array}{c}\text { Not affected number and } \\
\text { percentage }\end{array}$ & Total & Percentage \\
\hline Effect on work & $40(69 \%)$ & $18(31 \%)$ & 58 & $100 \%$ \\
\hline Off work* & $32(55 \%)$ & $26(45 \%)$ & 58 & $100 \%$ \\
\hline Reduced productivity & $21(36.2 \%)$ & $37(63.8 \%)$ & 58 & $100 \%$ \\
\hline
\end{tabular}




\begin{tabular}{|c|c|c|c|c|}
\hline Work dissatisfaction & $20(34.5 \%)$ & $38(65.5 \%)$ & 58 & $100 \%$ \\
\hline Effect on career progression & $38(65.5 \%)$ & $20(34.5 \%)$ & 58 & $100 \%$ \\
\hline
\end{tabular}

*1-4 days off work $=10(17.2 \%)$

a) The leiomyomata-related problems, $\chi 2=93.15, \mathrm{p}<0.001$ (Table 4)

Table 4: Leiomyomata-related problems and the symptoms' intensity Cross tabulation.

\begin{tabular}{|c|c|c|c|c|c|c|}
\hline & & & \multicolumn{3}{|c|}{ Symptoms Intensity } & \multirow{2}{*}{ Total } \\
\hline & & & Mild & Moderate & Severe & \\
\hline Symptoms Type & Effect on mode and energy & Count & 8 & 49 & 143 & 200 \\
\hline & & $\%$ within Symptoms Type & $4.00 \%$ & $24.50 \%$ & $71.50 \%$ & $100.00 \%$ \\
\hline & Effect on physical activity & Count & 13 & 54 & 133 & 200 \\
\hline & & $\%$ within Symptoms Type & $6.50 \%$ & $27.00 \%$ & $66.50 \%$ & $100.00 \%$ \\
\hline & Menstrual loss and duration & Count & 4 & 80 & 116 & 200 \\
\hline & & $\%$ within Symptoms Type & $2.00 \%$ & $40.00 \%$ & $58.00 \%$ & $100.00 \%$ \\
\hline & Pressure symptoms & Count & 21 & 78 & 101 & 200 \\
\hline & & $\%$ within Symptoms Type & $10.50 \%$ & $39.00 \%$ & $50.50 \%$ & $100.00 \%$ \\
\hline & Psychological impact & Count & 3 & 74 & 123 & 200 \\
\hline & & $\%$ within Symptoms Type & $1.50 \%$ & $37.00 \%$ & $61.50 \%$ & $100.00 \%$ \\
\hline & Sexual satisfaction & Count & 27 & 40 & 73 & 140 \\
\hline & & $\%$ within Symptoms Type & $19.30 \%$ & $28.60 \%$ & $52.10 \%$ & $100.00 \%$ \\
\hline & The sense of control & Count & 18 & 37 & 145 & 200 \\
\hline & & \% within Symptoms Type & $9.00 \%$ & $18.50 \%$ & $72.50 \%$ & $100.00 \%$ \\
\hline$T_{0}$ & & Count & 94 & 412 & 834 & 1340 \\
\hline 10 ld1 & & $\%$ within Symptoms Type & $7.00 \%$ & $30.70 \%$ & $62.20 \%$ & $100.00 \%$ \\
\hline
\end{tabular}

\begin{tabular}{|c|c|c|c|}
\hline & Value & df & Asymp. Sig. (2-sided) \\
\hline Pearson Chi-Square & $93.152 \mathrm{a}$ & 12 & 0 \\
\hline Likelihood Ratio & 91.288 & $1200.00 \%$ & $0.00 \%$ \\
\hline N of Valid Cases & 1340 & & \\
\hline
\end{tabular}

\section{Chi-Square Tests}

a. 0 cells $(0.0 \%)$ have expected count less than 5 . The minimum expected count is 9.82 .

Conclusion: We observed a strong association between the Leiomyomata-related problems and Symptoms Intensity, $\chi^{2}=93.15, \mathrm{p}$ $<0.001$

b) The physical activity, $\chi 2=317.03, \mathrm{p}<0.001$ (Tables 5 \& 6)

Table 5: Employment and symptoms' intensity Cross tabulation.

\begin{tabular}{|c|c|c|c|c|c|c|}
\hline & & & \multicolumn{2}{|c|}{ Symptom Intensity } & & Total \\
\hline & & & Mild symptoms & Moderate symptoms & Sever & oms \\
\hline \multirow{4}{*}{ Employment } & \multirow{2}{*}{ Employed } & \multirow{2}{*}{$\begin{array}{c}\text { Count } \\
\text { \% within group }\end{array}$} & $200.00 \%$ & $1400.00 \%$ & $4200.00 \%$ & 58 \\
\hline & & & $3.40 \%$ & $24.10 \%$ & $72.40 \%$ & $100.00 \%$ \\
\hline & \multirow{2}{*}{ Housewives } & \multirow{2}{*}{$\begin{array}{l}\text { Count } \\
\text { \% within group }\end{array}$} & $200.00 \%$ & $5900.00 \%$ & $8100.00 \%$ & 142 \\
\hline & & & $1.40 \%$ & $41.50 \%$ & $57.00 \%$ & $100.00 \%$ \\
\hline \multirow{2}{*}{ Total } & & \multirow{2}{*}{$\begin{array}{l}\text { Count } \\
\% \text { of total }\end{array}$} & $400.00 \%$ & $7300.00 \%$ & \#\#\#\#\#\#\#\# & 200 \\
\hline & & & $2.00 \%$ & $36.50 \%$ & $61.50 \%$ & $100.00 \%$ \\
\hline
\end{tabular}




\begin{tabular}{|c|c|c|c|}
\hline & Value & df & Asymp. Sig. (2-sided) \\
\hline Pearson Chi-Square & $5.859 \mathrm{a}$ & 2 & 0.053 \\
\hline Likelihood Ratio & 6.019 & $200.00 \%$ & $4.90 \%$ \\
\hline N of Valid Cases & 200 & & \\
\hline
\end{tabular}

\section{Chi-Square Tests}

a. 2 cells $(33.3 \%)$ have expected count less than 5 . The minimum expected count is 1.16 .

Conclusion: We didn't observe association between the Employment and Symptom Intensity, $\chi 2=5.86, p>0.05$

Table 6: Physical Activity and symptoms' intensity Cross tabulation.

\begin{tabular}{|c|c|c|c|c|c|c|}
\hline & & & \multicolumn{3}{|c|}{ Symptom Intensity } & \multirow[b]{2}{*}{ Total } \\
\hline & & & Mild symptoms & $\begin{array}{l}\text { Moderate } \\
\text { symptoms }\end{array}$ & Severe symptoms & \\
\hline \multirow[t]{6}{*}{ Physical Activity } & Full activity & Count & $0.00 \%$ & $500.00 \%$ & \#\#\#\#\#\#\#\# & 110 \\
\hline & & $\begin{array}{c}\% \text { within Physical } \\
\text { Activity }\end{array}$ & $0.00 \%$ & $4.50 \%$ & $95.50 \%$ & $100.00 \%$ \\
\hline & Mild activity & Count & $400.00 \%$ & $0.00 \%$ & $0.00 \%$ & 4 \\
\hline & & $\begin{array}{c}\% \text { within Physical } \\
\text { Activity }\end{array}$ & $100.00 \%$ & $0.00 \%$ & $0.00 \%$ & $100.00 \%$ \\
\hline & Moderate activity & Count & $0.00 \%$ & $6800.00 \%$ & $1800.00 \%$ & 86 \\
\hline & & $\begin{array}{c}\% \text { within Physical } \\
\text { Activity }\end{array}$ & $0.00 \%$ & $79.10 \%$ & $20.90 \%$ & $100.00 \%$ \\
\hline \multirow[t]{2}{*}{ Total } & & Count & $400.00 \%$ & $7300.00 \%$ & \#\#\#\#\#\#\#\# & 200 \\
\hline & & $\begin{array}{c}\text { \% within Physical } \\
\text { Activity }\end{array}$ & $2.00 \%$ & $36.50 \%$ & $61.50 \%$ & $100.00 \%$ \\
\hline
\end{tabular}

\begin{tabular}{|c|c|c|c|}
\hline & Value & df & Asymp. Sig. (2-sided) \\
\hline Pearson Chi-Square & $317.028 \mathrm{a}$ & 4 & 0 \\
\hline Likelihood Ratio & 169.111 & $400.00 \%$ & $0.00 \%$ \\
\hline N of Valid Cases & 200 & & \\
\hline
\end{tabular}

Chi-Square Tests

a. 5 cells $(55.6 \%)$ have expected count less than 5 . The minimum expected count is .08 .

Conclusion: We observed a strong association between Physical Activity and Symptom Intensity, $\chi^{2}=317.03, p<0.001$

c) The mode and energy, $\chi 2=149.69, \mathrm{p}<0.001$ (Table 7)

Table 7: Mode and Energy and symptoms' intensity Cross tabulation.

\begin{tabular}{|c|c|c|c|c|c|}
\hline & & \multicolumn{3}{|c|}{ Symptom Intensity } & \multirow{2}{*}{ Total } \\
\hline & & Mild symptoms & Moderate symptoms & Severe symptoms & \\
\hline Mode and Energy & Count & $200.00 \%$ & $1100.00 \%$ & $0.00 \%$ & 13 \\
\hline $\begin{array}{l}\text { Mild affected mode } \\
\text { and energy }\end{array}$ & $\begin{array}{l}\text { \% within Mode and } \\
\text { Energy }\end{array}$ & $15.40 \%$ & $84.60 \%$ & $0.00 \%$ & $100.00 \%$ \\
\hline \multirow{2}{*}{$\begin{array}{l}\text { Moderate affected } \\
\text { mode and energy }\end{array}$} & Count & $200.00 \%$ & $5000.00 \%$ & $300.00 \%$ & 55 \\
\hline & $\begin{array}{c}\text { \% within Mode and } \\
\text { Energy }\end{array}$ & $3.60 \%$ & $90.90 \%$ & $5.50 \%$ & $100.00 \%$ \\
\hline \multirow{2}{*}{$\begin{array}{l}\text { Severe affected mode } \\
\text { and energy }\end{array}$} & Count & $0.00 \%$ & $1200.00 \%$ & \#\#\#\#\#\#\#\# & 132 \\
\hline & $\begin{array}{l}\% \text { within Mode and } \\
\text { Energy }\end{array}$ & $0.00 \%$ & $9.10 \%$ & $90.90 \%$ & $100.00 \%$ \\
\hline \multirow[b]{2}{*}{ Total } & Count & $400.00 \%$ & $7300.00 \%$ & \#\#\#\#\#\#\#\# & 200 \\
\hline & $\begin{array}{l}\% \text { within Mode and } \\
\text { Energy }\end{array}$ & $2.00 \%$ & $36.50 \%$ & $61.50 \%$ & $100.00 \%$ \\
\hline
\end{tabular}




\begin{tabular}{|c|c|c|c|}
\hline & Value & df & Asymp. Sig. (2-sided) \\
\hline Pearson Chi-Square & $149.693 \mathrm{a}$ & 4 & 0 \\
\hline Likelihood Ratio & 166.206 & $400.00 \%$ & $0.00 \%$ \\
\hline N of Valid Cases & 200 & & \\
\hline
\end{tabular}

\section{Chi-Square Tests}

a. 4 cells $(44.4 \%)$ have expected count less than 5 . The minimum expected count is .26 .

Conclusion: We observed a strong association between Mode and Energy and Symptom Intensity, $\chi 2=149.69, \mathrm{p}<0.001$

d) The sense of control, $\chi 2=122.03, \mathrm{p}<0.001$ (Table 8)

Table 8: Sense of control and symptoms' intensity Cross tabulation.

\begin{tabular}{|c|c|c|c|c|c|c|}
\hline & & & \multicolumn{3}{|c|}{ Symptom Intensity } & \multirow[b]{2}{*}{ Total } \\
\hline & & & Mild symptoms & $\begin{array}{c}\text { Moderate } \\
\text { symptoms }\end{array}$ & Severe symptoms & \\
\hline Sense of control & $\begin{array}{l}\text { Mildly affected } \\
\text { sense of control }\end{array}$ & Count & $200.00 \%$ & $1600.00 \%$ & $0.00 \%$ & $1800.00 \%$ \\
\hline & & $\begin{array}{c}\% \text { within Sense of } \\
\text { control }\end{array}$ & $11.10 \%$ & $88.90 \%$ & $0.00 \%$ & $100.00 \%$ \\
\hline \multicolumn{2}{|c|}{ Moderately affected sense of control } & Count & $200.00 \%$ & $3400.00 \%$ & $0.00 \%$ & $3600.00 \%$ \\
\hline & & $\begin{array}{c}\% \text { within Sense of } \\
\text { control }\end{array}$ & $5.60 \%$ & $94.40 \%$ & $0.00 \%$ & $100.00 \%$ \\
\hline \multicolumn{2}{|c|}{ Severely affected sense of control } & Count & $0.00 \%$ & $2300.00 \%$ & \#\#\#\#\#\#\#\# & \#\#\#\#\#\#\#\# \\
\hline & & $\begin{array}{c}\% \text { within Sense of } \\
\text { control }\end{array}$ & $0.00 \%$ & $15.80 \%$ & $84.20 \%$ & $100.00 \%$ \\
\hline \multicolumn{2}{|c|}{ Total } & Count & $400.00 \%$ & $7300.00 \%$ & \#\#\#\#\#\#\#\# & \#\#\#\#\#\#\#\# \\
\hline & & $\begin{array}{c}\% \text { within Sense of } \\
\text { control }\end{array}$ & $2.00 \%$ & $36.50 \%$ & $61.50 \%$ & $100.00 \%$ \\
\hline
\end{tabular}

\begin{tabular}{|c|c|c|c|}
\hline & Value & df & Asymp. Sig. (2-sided) \\
\hline Pearson Chi-Square & $122.027 \mathrm{a}$ & 4 & 0 \\
\hline Likelihood Ratio & 142.843 & $400.00 \%$ & $0.00 \%$ \\
\hline N of Valid Cases & 200 & & \\
\hline
\end{tabular}

\section{Chi-Square Tests}

a. 3 cells $(33.3 \%)$ have expected count less than 5 . The minimum expected count is .36 .

Conclusion: We observed a strong association between Sense of control * Symptom Intensity, $\chi^{2}=122.03, p<0.001$

e) Sexual dissatisfaction, $\chi 2=79.07, \mathrm{p}<0.001$ (Table 9)

Table 9: Sexual Dissatisfaction and symptoms' intensity Cross tabulation.

\begin{tabular}{|c|c|c|c|c|c|}
\hline \multirow{4}{*}{$\begin{array}{c}\text { Sexual Dissatisfaction } \\
\text { Mild sexual } \\
\text { dissatisfaction }\end{array}$} & \multirow[b]{3}{*}{ Count } & \multicolumn{2}{|c|}{ Symptom Intensity } & \multirow{2}{*}{\multicolumn{2}{|c|}{$\begin{array}{c}\text { Total } \\
\text { Severe symptoms }\end{array}$}} \\
\hline & & \multirow{2}{*}{$\begin{array}{c}\text { Mild symptoms } \\
300.00 \% \\
\end{array}$} & \multirow{2}{*}{$\begin{array}{c}\text { Moderate symptoms } \\
2400.00 \% \\
\end{array}$} & & \\
\hline & & & & $0.00 \%$ & 27 \\
\hline & $\begin{array}{l}\% \text { within Sexual } \\
\text { Dissatisfaction }\end{array}$ & $11.10 \%$ & $88.90 \%$ & $0.00 \%$ & $100.00 \%$ \\
\hline \multirow{2}{*}{$\begin{array}{l}\text { Moderate sexual } \\
\text { dissatisfaction }\end{array}$} & Count & $0.00 \%$ & $1900.00 \%$ & $2100.00 \%$ & $4000.00 \%$ \\
\hline & $\begin{array}{l}\text { \% within Sexual } \\
\text { Dissatisfaction }\end{array}$ & $0.00 \%$ & $47.50 \%$ & $52.50 \%$ & $100.00 \%$ \\
\hline \multirow{2}{*}{$\begin{array}{r}\text { Severe sexual } \\
\text { dissatisfaction }\end{array}$} & Count & $0.00 \%$ & $600.00 \%$ & $6700.00 \%$ & $7300.00 \%$ \\
\hline & $\begin{array}{l}\% \text { within Sexual } \\
\text { Dissatisfaction }\end{array}$ & $0.00 \%$ & $8.20 \%$ & $91.80 \%$ & $100.00 \%$ \\
\hline
\end{tabular}




\begin{tabular}{|c|c|c|c|c|c|}
\hline \multirow{3}{*}{ Total } & Count & $300.00 \%$ & $4900.00 \%$ & $8800.00 \%$ & \#\#\#\#\#\#\#\# \\
\cline { 2 - 6 } & $\begin{array}{c}\text { \% within Sexual } \\
\text { Dissatisfaction }\end{array}$ & $2.10 \%$ & $35.00 \%$ & $62.90 \%$ & $100.00 \%$ \\
\hline
\end{tabular}

\begin{tabular}{|c|c|c|c|}
\hline & Value & df & Asymp. Sig. (2-sided) \\
\hline Pearson Chi-Square & $79.072 \mathrm{a}$ & 4 & 0 \\
\hline Likelihood Ratio & 91.993 & $400.00 \%$ & $0.00 \%$ \\
\hline N of Valid Cases & 140 & & \\
\hline
\end{tabular}

\section{Chi-Square Tests}

a. 3 cells $(33.3 \%)$ have expected count less than 5. The minimum expected count is .58.

Conclusion: We observed a strong association between Sexual Dissatisfaction and Symptom Intensity, $\chi^{2}=79.07, \mathrm{p}<0.001$

f) The socio-psychological well-being, $\chi 2=317.03, \mathrm{p}<0.001$ (Table 10)

Table 10: Socio-psychological well-being and symptoms' intensity Cross tabulation.

\begin{tabular}{|c|c|c|c|c|c|}
\hline \multirow{4}{*}{$\begin{array}{l}\text { Socio-psychological } \\
\text { Wellbeing Mildly- } \\
\text { affected socio- } \\
\text { psychological well } \\
\text { being }\end{array}$} & \multirow[b]{3}{*}{ Count } & \multicolumn{2}{|c|}{ Symptom Intensity } & \multirow{2}{*}{\multicolumn{2}{|c|}{$\begin{array}{c}\text { Total } \\
\text { Severe symptoms }\end{array}$}} \\
\hline & & \multirow{2}{*}{$\begin{array}{c}\text { Mild symptoms } \\
400.00 \% \\
\end{array}$} & \multirow{2}{*}{$\begin{array}{c}\text { Moderate symptoms } \\
0.00 \% \\
\end{array}$} & & \\
\hline & & & & $0.00 \%$ & 4 \\
\hline & $\begin{array}{l}\% \text { within Socio- } \\
\text { psychological Well } \\
\text { being }\end{array}$ & $100.00 \%$ & $0.00 \%$ & $0.00 \%$ & $100.00 \%$ \\
\hline \multirow[b]{2}{*}{$\begin{array}{l}\text { Moderately-affected } \\
\text { psychological well } \\
\text { being }\end{array}$} & Count & $0.00 \%$ & $6800.00 \%$ & $1800.00 \%$ & $8600.00 \%$ \\
\hline & $\begin{array}{l}\text { \% within Socio- } \\
\text { psychological Well } \\
\text { being }\end{array}$ & $0.00 \%$ & $79.10 \%$ & $20.90 \%$ & $100.00 \%$ \\
\hline \multirow[b]{2}{*}{$\begin{array}{l}\text { Severely-affected } \\
\text { psychological well } \\
\text { being }\end{array}$} & Count & $0.00 \%$ & $500.00 \%$ & \#\#\#\#\#\#\#\# & \#\#\#\#\#\#\#\# \\
\hline & $\begin{array}{c}\% \text { within Socio- } \\
\text { psychological Well } \\
\text { being }\end{array}$ & $0.00 \%$ & $4.50 \%$ & $95.50 \%$ & $100.00 \%$ \\
\hline \multirow[b]{2}{*}{ Total } & Count & $400.00 \%$ & $7300.00 \%$ & \#\#\#\#\#\#\#\# & \#\#\#\#\#\#\#\# \\
\hline & $\begin{array}{l}\% \text { within Socio- } \\
\text { psychological Well } \\
\text { being }\end{array}$ & $2.00 \%$ & $36.50 \%$ & $61.50 \%$ & $100.00 \%$ \\
\hline
\end{tabular}

\begin{tabular}{|c|c|c|c|}
\hline & Value & df & Asymp. Sig. (2-sided) \\
\hline Pearson Chi-Square & $317.028 \mathrm{a}$ & 4 & 0 \\
\hline Likelihood Ratio & 169.111 & $400.00 \%$ & $0.00 \%$ \\
\hline N of Valid Cases & 200 & & \\
\hline
\end{tabular}

\section{Chi-Square Tests}

a. 5 cells $(55.6 \%)$ have expected count less than 5 . The minimum expected count is .08 .

Conclusion: We observed a strong association between Socio-psychological Well-being and Symptom Intensity, $\chi 2=317.03, \mathrm{p}<0.001$

g) The psychological well-being, $\chi 2=144.94, \mathrm{p}<0.001$ (Table 11)

Table 11: Psychological Well-being and symptoms' intensity Cross tabulation.

\begin{tabular}{|c|c|c|c|c|c|}
\hline \multicolumn{2}{|c|}{} & \multicolumn{2}{c|}{ Symptom Intensity } & \multicolumn{2}{c|}{ Total } \\
\cline { 3 - 5 } \multicolumn{2}{|c|}{} & Mild Symptoms & Moderate Symptoms & \multicolumn{2}{c|}{ Severe Symptoms } \\
\hline $\begin{array}{c}\text { Psychological Well being } \\
\text { Mildly-affected psychological well } \\
\text { being }\end{array}$ & Count & $200.00 \%$ & $700.00 \%$ & $0.00 \%$ & 9 \\
\cline { 2 - 5 } & $\begin{array}{c}\text { \% within Psychological } \\
\text { Well being }\end{array}$ & $22.20 \%$ & $77.80 \%$ & $0.00 \%$ & $100.00 \%$ \\
\hline
\end{tabular}




\begin{tabular}{|c|c|c|c|c|c|}
\hline \multirow{2}{*}{$\begin{array}{l}\text { Moderately-affected psychological } \\
\text { well being }\end{array}$} & Count & $200.00 \%$ & $4700.00 \%$ & $0.00 \%$ & $4900.00 \%$ \\
\hline & $\begin{array}{c}\% \text { within Psychological } \\
\text { Well being }\end{array}$ & $4.10 \%$ & $95.90 \%$ & $0.00 \%$ & $100.00 \%$ \\
\hline \multirow{2}{*}{$\begin{array}{l}\text { Severely-affected psychological well } \\
\text { being }\end{array}$} & Count & $0.00 \%$ & $1900.00 \%$ & \#\#\#\#\#\#\#\# & \#\#\#\#\#\#\#\# \\
\hline & $\begin{array}{c}\% \text { within Psychological } \\
\text { Well being }\end{array}$ & $0.00 \%$ & $13.40 \%$ & $86.60 \%$ & $100.00 \%$ \\
\hline \multirow[b]{2}{*}{ Total } & Count & $400.00 \%$ & $7300.00 \%$ & \#\#\#\#\#\#\#\# & \#\#\#\#\#\#\#\# \\
\hline & $\begin{array}{c}\% \text { within Psychological } \\
\text { Well being }\end{array}$ & $2.00 \%$ & $36.50 \%$ & $61.50 \%$ & $100.00 \%$ \\
\hline
\end{tabular}

\begin{tabular}{|c|c|c|c|}
\hline & Value & df & Asymp. Sig. (2-sided) \\
\hline Pearson Chi-Square & $144.936 \mathrm{a}$ & 4 & 0 \\
\hline Likelihood Ratio & 160.017 & $400.00 \%$ & $0.00 \%$ \\
\hline N of Valid Cases & 200 & & \\
\hline
\end{tabular}

\section{Chi-Square Tests}

a. 4 cells $(44.4 \%)$ have expected count less than 5 . The minimum expected count is .18 .

Conclusion: We observed a strong association between Psychological Well-being and Symptom Intensity, $\chi^{2}=144.94, p<0.001$

h) The social engagement, $\chi 2=142.54, \mathrm{p}<0.001$ (Table 12)

Table 12: Social Engagement and symptoms' intensity Cross tabulation.

\begin{tabular}{|c|c|c|c|c|c|}
\hline \multirow{4}{*}{$\begin{array}{c}\text { Social Engagement } \\
\text { Mildly-affected social } \\
\text { engagement }\end{array}$} & \multirow[b]{3}{*}{ Count } & \multicolumn{2}{|c|}{ Symptom Intensity } & \multirow{2}{*}{\multicolumn{2}{|c|}{$\begin{array}{c}\text { Total } \\
\text { Severe symptoms }\end{array}$}} \\
\hline & & \multirow{2}{*}{$\begin{array}{c}\text { Mild symptoms } \\
200.00 \%\end{array}$} & \multirow{2}{*}{$\begin{array}{c}\text { Moderate symptoms } \\
1100.00 \%\end{array}$} & & \\
\hline & & & & $0.00 \%$ & 13 \\
\hline & \% within Social Engagement & $15.40 \%$ & $84.60 \%$ & $0.00 \%$ & $100.00 \%$ \\
\hline \multirow{2}{*}{$\begin{array}{l}\text { Moderately-affected } \\
\text { social engagement }\end{array}$} & Count & $200.00 \%$ & $4900.00 \%$ & $400.00 \%$ & $5500.00 \%$ \\
\hline & \% within Social Engagement & $3.60 \%$ & $89.10 \%$ & $7.30 \%$ & $100.00 \%$ \\
\hline \multirow{2}{*}{$\begin{array}{c}\text { Severely-affected } \\
\text { social engagement }\end{array}$} & Count & $0.00 \%$ & $1300.00 \%$ & \#\#\#\#\#\#\#\# & \#\#\#\#\#\#\#\# \\
\hline & \% within Social Engagement & $0.00 \%$ & $9.80 \%$ & $90.20 \%$ & $100.00 \%$ \\
\hline \multirow{2}{*}{ Total } & Count & $400.00 \%$ & $7300.00 \%$ & \#\#\#\#\#\#\#\# & \#\#\#\#\#\#\#\# \\
\hline & \% within Social Engagement & $2.00 \%$ & $36.50 \%$ & $61.50 \%$ & $100.00 \%$ \\
\hline
\end{tabular}

\begin{tabular}{|c|c|c|c|}
\hline & Value & df & Asymp. Sig. (2-sided) \\
\hline Pearson Chi-Square & $142.543 \mathrm{a}$ & 4 & 0 \\
\hline Likelihood Ratio & 156.385 & $400.00 \%$ & $0.00 \%$ \\
\hline N of Valid Cases & 200 & & \\
\hline
\end{tabular}

\section{Chi-Square Tests}

a. 4 cells $(44.4 \%)$ have expected count less than 5 . The minimum expected count is .26.

Conclusion: We observed a strong association between Social Engagement and Symptom Intensity, $\chi^{2}=142.54, p<0.001$

i) The woman's concern about the leiomyomata, $\chi 2=130.17, \mathrm{p}<0.001$ (Table 13)

Table 13: Concern and symptoms' intensity Cross tabulations.

\begin{tabular}{|c|c|c|c|c|c|}
\hline \multicolumn{2}{|c|}{} & \multicolumn{2}{|c|}{ Symptom Intensity } & \multicolumn{2}{|c|}{ Total } \\
\cline { 2 - 6 } & & Mild symptoms & Moderate symptoms & \multicolumn{2}{|c|}{ Severe symptoms } \\
\hline \multirow{2}{*}{$\begin{array}{c}\text { Concern } \\
\text { Mild concern }\end{array}$} & Count & $200.00 \%$ & $1100.00 \%$ & $0.00 \%$ & 13 \\
\cline { 2 - 6 } & \% within Concern & $15.40 \%$ & $84.60 \%$ & $0.00 \%$ & $100.00 \%$ \\
\hline
\end{tabular}




\begin{tabular}{|c|c|c|c|c|c|}
\hline \multirow{2}{*}{ Moderate concern } & Count & $200.00 \%$ & $4300.00 \%$ & $200.00 \%$ & $4700.00 \%$ \\
\cline { 2 - 6 } & \% within Concern & $4.30 \%$ & $91.50 \%$ & $4.30 \%$ & $100.00 \%$ \\
\hline \multirow{2}{*}{ Severe concern } & Count & $0.00 \%$ & $1900.00 \%$ & \#\#\#\#\#\#\#\# & \#\#\#\#\#\#\#\# \\
\cline { 2 - 6 } & \% within Concern & $0.00 \%$ & $13.60 \%$ & \#\#\#\#\#\#\#\# & \#\#\#\#\#\#\#\# \\
\hline \multirow{2}{*}{ Total } & Count & $400.00 \%$ & $7300.00 \%$ & $61.50 \%$ & $1000.00 \%$ \\
\cline { 2 - 6 } & \% within Concern & $2.00 \%$ & $36.50 \%$ & & $10 \%$ \\
\hline
\end{tabular}

\begin{tabular}{|c|c|c|c|}
\hline & Value & df & Asymp. Sig. (2-sided) \\
\hline Pearson Chi-Square & $130.172 \mathrm{a}$ & 4 & 0 \\
\hline Likelihood Ratio & 142.774 & $400.00 \%$ & $0.00 \%$ \\
\hline N of Valid Cases & 200 & & \\
\hline
\end{tabular}

\section{Chi-Square Tests}

a. 4 cells $(44.4 \%)$ have expected count less than 5. The minimum expected count is 26 .

Conclusion: We observed a strong association between Concern and Symptom Intensity, $\chi 2=130.17, \mathrm{p}<0.001$

There was no observed association between the employment and the symptoms' intensity, $\chi 2=5.86, \mathrm{p}>0.05$ (Table 5).

\section{Discussion}

The study has shown that the incidence of Uterine Leiomyomata (UL) is more common (93.6\%) among women of child-bearing age (20-39 years), this is in accordance with the observation that UL is prevalent in the third and fourth decade of life and one of the most common benign tumors in this age group and is more common among black women $[5,4,6,33,34,35]$. All the participants attained some level of education and $70 \%$ had secondary-school or higher education and this might have allowed them to have a better in depth understanding of the disease and its related problems. 66 (33\%) the women in the study had complications that could be attributed to the UL such as; infertility, miscarriage and anemia. The miscarriage rate in this study is $19.8 \%$. The association between UL and adverse reproductive outcomes was observed in several studies $[28,29,14,3,30,27,17,36]$, and it has been hypothesized that the compressed endometrial vascular supply by the leiomyomata may affect the fetus adversely resulting in miscarriage [36,37]. Many studies attributed heavy menstrual loss to UL [38,12,13,39]; however, a case study by Bachmann and coworkers [40] did not find a clinical support to explain anemia in women with submucosal UL, and in our study, we observed a lower rate of anemia of $5 \%$, compared to reported rates by Noor and coworkers (2009). The possible explanation for this discrepancy is that perhaps our study population is probably better nurtured as inferred from their: level of education, possible higher income and enhanced social status. The most commonly reported symptom in relation to UL is severe prolonged heavy menstrual loss followed by pressure symptoms [12]. The figures from our study have strongly shown the association between the problems-related to the leiomyomata and different aspects of the women activities and wellbeing and are consistent with other the findings [13]. Zimmermann and colleagues surveyed 21476 women with UL from 8 countries and reported heavy menstrual bleeding and pressure symptoms in
$58.9 \%$ and $32.6 \%$ of the women respectively. Like our study, these symptoms have variable impacts on the women's activities; some were mildly affected (6.4\%), while the effect was either moderate $(27.2 \%)$ or severe $(66.4 \%)$. Other quantitative studies have shown that UL symptoms have a substantial negative impact on the QOL and physical activities of the women with severe symptoms. $71.2 \%$ of the women in our study have reported that they lost energy, or the UL have affected their activities moderately (24.8\%) or mildly $(4 \%)$.

Our study has observed a strong association between the intensity of the symptoms and the: leiomyomata-related problems, physical activity, psychological, socio-psychological, mode and energy. These effects are very likely to have an impact on the women's activities and performance. 58 (29\%) employed women claimed that the UL had negative impact on: their work, performance and productivity and 55\% were off work due to the symptoms attributed to UL; $17.2 \%$ were absent from work for 1-4 days, and nearly two third (65.5\%) of the employed women were dissatisfied with their career progression and associated that with UL-related symptoms. Borah and his coworkers (2013) studied 1756 women from different countries and found that the career of $36.1 \%$ of the employed women was affected by the UL, $24 \%$ failed to fulfil their career potentials and $28 \%$ had reduced productivity by missing work. Most of the participants in our study have expressed concern regarding the effect of UL on their fertility, which could probably explain their stress and anxiety and its effect on marital and social lives and work with potential loss of revenue and income. Some women were worried about their body image, especially single and younger women, and some of them felt embarrassed by the symptoms and many of the latter group feared that UL may lessen their chance of getting married. In our study we observed that $76.7 \%$ of the parous women are of low parity, but we do not have robust evidence to associate this with UL, but conversely, we could 
not associate it with any other factors. On the other hand, a metaanalysis of 23 studies on the effect of uterine UL on women fertility found that UL in general, regardless of location, were associated with a $15 \%$ reduction in pregnancy rates and $30 \%$ reduction in live birth rates [16] and another study by Ben-Nagi, coworkers [41] reported an association between UL and subfertility; however, this association might be not fully understood and controversial [42]. In our study 127 (63.5\%) of the participants are nulliparous and 38 (19\%) of all participants have a history of subfertility and certainly the rate would have been even higher (29.5\%), if the demonolater is restricted to the 129 married women.

Although leiomyomata are one of the most common benign tumors that affect reproductive-age women, there are only few studies that have assessed in depth the effect of the tumor on the women's reproduction. There might be an argument that a cause and effect relationship could be the case between submucosal UL and reproductive failure via the disturbance of the endometrial implantation interface; however, UL situated in sites not interfering with transport of the germ cells or blastocyst implantation or fetal passage through the birth canal may have little or no impact on reproduction performance as evident from the many women with UL of different sizes and locations who achieved their reproduction potentials without any complications. Many women (83.6\%) in our study thought that heavy prolonged menstrual loss and pelvic pain have limited their physical, social and household activities such as; cooking, shopping, cleaning, and childcare and they worried about their effect on their family and marital life and most of them felt that they lost the control of their life and claimed that their social life activities were negatively affected to a moderate degree, while $80.6 \%$ of women stated that their sexual life was moderately to severely affect by the UL. Our study observed strong association between the intensity of the symptoms and the: sense of control and sexual dissatisfaction. Few studies have examined the effect of UL on women 's sexual satisfaction, and even fewer assessed the effects of different treatment modalities on the women's sexual satisfaction [Ferrero et al, 2006]. It is worth noting that in our study 6 out of the 11 (54.4\%) divorcee claimed that the leiomyomata have affected their sexual and marital lives negatively and could have contributed to their marital disharmony and divorce. A controlledclinical study by Ertunc and coworkers (2009) compared the effect of UL on sexuality of affected women against a control of women without UL and found that UL appear to interfere with sexual satisfaction mainly due to dyspareunia, although they do not seem to have effect on libido, arousal, lubrication or orgasm.

Our study has observed that the intensity of the symptoms is strongly associated with negative impact on the woman's well-being and life. However, the association may not be as straightforward as we think and further studies to evaluate the complexity and the impact of UL on daily life of the women is required. Many women felt that UL controls and restricts their lives, leaving them powerless.
The findings regarding the incidence of dyspareunia vary but suggest that it has a significant social and psychological impact, but little is known about the impact of UL on the partners and children or the wider family. Limited research suggests that these social and sexual impact causes worry, depression and feelings of inadequacy in our study we found that $94 \%$ of the participants have moderate to severe psychological concerns and we also observed strong association with the intensity of the symptoms and that included the: socio-psychological well-being, social engagement and loss on control. Complaints such as: emotional distress, hopelessness, isolation, worthlessness and depression are not uncommon and feeling miserable, depressed, moody and short tempered are frequently mentioned. Most of the participants worried about treatment duration and prognosis, especially the surgical modalities like hysterectomy. Borah and coworkers (2012) studied 1000 women in the USA diagnosed with UL and assessed the psychological concerns of the women, most of them reported: fears that the UL may grow (79\%), there is something inside of them that does not belong there (69\%), they would experience future health complications (63\%), they will need a hysterectomy (55\%), and that UL would turn into cancer (54\%). Few studies addressed the QOL with regard to UL and there is a lack of psychometrically sound disease-specific HRQOL instrumentation. Furthermore, many of the available studies were uncontrolled or retrospective in nature. In many cases, a number of different benign gynecological conditions were studied along with UL, leading to an inability to make explicit inferences regarding HRQOL related to UL alone. It remains a challenge for future studies to address these issues and have a better in depth understanding of the QOL consequences of UL. The management of UL has financial consequences on the: healthcare system, work productivity, women' mental wellbeing, and family life. For these reasons more research is needed.

\section{Conclusion}

UL are common during the child-bearing age and might be contributing to the inability of a large proportion of the women to fulfil their ambition of having a family. UL appear to have a significant social and psychological impact on the lives of women across several domains. UL are possible cause of heavy menstrual periods and menstrual pain and might have a negative impact on women's quality of life, influencing there: sexual, social and work life. The effect of the UL on the women's health and quality of life and the rest of her family is still limited and further research is required. Health providers should consider the impact of treatment modalities when counseling women, as they have a significant influence on the women's abilities. Health professionals should educate and empower women with UL to enable women to have a better understanding of the disease and enable them to make informed decisions regarding their management. Increasing the awareness of the disease among the general women's population should consider the utilization all the available media including 
the social media, which has become part of the daily life of many people. The underlying aetiology of UL deserves further work, especially among the black women population who apparently have a disproportionately higher incidence of the disease. The treatment options for leiomyomata deserve further appraisal as it appears to have a considerable effect on the women's HRQL. Research on the impact of UL on the wider family is required. We think a prospective randomized case-control study, which is community based including a wider population is more likely to shed more light on the impact of UL on the women's QOL and on her surrounding community. Furthermore, there is an urgent need to evaluate and or develop support for the women with UL and their families, because this chronic disease could be debilitating to the whole family both socially and economically.

\section{Acknowledgement}

We are grateful to all the participants who made this work possible and we grateful to the colleagues who helped in this research.

\section{References}

1. Kempson RL, Hendrickson MR (2000) Smooth muscle, endometrial stromal, and mixed Müllerian tumors of the uterus. Mod Pathol 13(3): 328-342.

2. Leppert PC, Catherino WH, Segars JH (2006) A new hypothesis about the origin of uterine fibroids based on gene expression profiling with microarrays. Am J Obstet Gynecol 195(2): 415-420.

3. Parker WH (2007) Etiology, symptomatology, and diagnosis of uterine myomas. Fertil Steril 87(4): 725-736.

4. Stewart EA (2001) Uterine Fibroids. Lancet 357(9252): 293-298.

5. Marshall LM, Spiegelman D, Barbieri RL, Goldman MB, Manson JE, et al. (1997) Variation in the incidence of uterine leiomyoma among premenopausal women by age and race. Obstet Gynecol 90(6): 967-973.

6. Baird DD, Dunson DB, Hill MC, Cousins D, Schectman JM (2003) High cumulative incidence of uterine leiomyoma in black and white women: Ultrasound evidence. Am J Obstet Gynecol 188(1): 100-107.

7. Rein MS, Barbieri RL, Friedman AJ (1995) Progesterone: a critical role in the pathogenesis of uterine myomas. Am J Obstet Gynecol 172(1 Pt 1): $14-18$.

8. Tomlinson IPM, Alam NA, Rowan AJ, Barclay E, Kelsell D, et al. (2002) Germline mutations in the fumarate hydratase gene predispose to dominantly inherited uterine fibroids, skin leiomyomata and renal cell cancer. Nature Genetics 30(4): 406-10

9. Wise LA, Palmer JR, Harlow BL, Spiegelman D, Stewart EA, et al. (2004) Risk of uterine leiomyomata in relation to tobacco, alcohol and caffeine consumption in the Black Women's Health Study. Hum Reprod 19(8): 1746-1754

10. Mehine $M$, Kaasinen E, Heinonen H-R, Mäkinen N, Kämpjärvi K, et al. (2016) Integrated data analysis reveals uterine leiomyoma subtypes with distinct driver pathways and biomarkers. Proc Natl Acad Sci USA 113(5):1315-1320.

11. Mehine M, Kaasinen E, Mäkinen N, Katainen R, Kämpjärvi K, et al. (2013) Characterization of Uterine Leiomyomas by Whole Genome Sequencing. New England Journal of Medicine 369(1): 43-53.

12. Wegienka G, Baird DD, Hertz-Picciotto I, Harlow SD, Steege JF, et al. (2003) Self-reported heavy bleeding associated with uterine leiomyomata. Obstet Gynecol 101(3): 431-437.
13.Zimmermann A, Bernuit D, Gerlinger C, Schaefers M, Geppert K (2012) Prevalence, symptoms and management of uterine fibroids: an international internet-based survey of 21,746 women. BMC Women's Health 12: 6 .

14. Qidwai G, Caughey AB, Jacoby AF (2006) Obstetric outcomes in women with sonographically identified uterine leiomyomata. Obstet Gynecol 107(2 Part 1):376-82.

15. Klatsky PC, Tran ND, Caughey AB, Fujimoto VY (2008) Fibroids and reproductive outcomes: a systematic literature review from conception to delivery. Am J Obstet Gynecol 198(4): 357-366.

16. Pritts EA, Parker WH, Olive DL (2009) Fibroids and infertility: An updated systematic review of the evidence. Fertil Steril 91(4): 12151223.

17. Navid S, Arshad S, Qurat-ul-Ain, Meo RA (2012) Impact of Leiomyoma in Pregnancy. J Ayub Med CollAbbottabad 24(1): 90-92.

18. Stewart EA, Nicholson WK, Bradley L, Borah BJ (2013) The burden of uterine fibroids for African-American women: results of a national survey. J Womens Health (Larchmt) 22(10): 807-816.

19. Ertunc D, Uzun R, Tok EC, Doruk A, Dilek S (2009) The Effect of Myoma Uteri and Myomectomy on Sexual Function. Journal of Sexual Medicine 6(4): 1032-1038.

20. Lippman SA, Warner M, Samuels S, Olive D, Vercellini P, et al. (2003) Uterine fibroids and gynecologic pain symptoms in a population-based study. Fertil Steril 80(6):1488-1494.

21. Ferrero S, Abbamonte LH, Giordano M, Parisi M, Ragni N, et al. (2006) Uterine myomas, dyspareunia, and sexual function. Fertil Steril 86(5): 1504-1510.

22. Borah BJ, Nicholson WK, Bradley L, Stewart EA (2013) The impact of uterine leiomyomas: a national survey of affected women. Am J Obstet Gynecol 209(4): 319.e1-319.e20.

23. Wilcox LS, Koonin LM, Pokras R, Strauss LT, Xia Z, et al. (1994) Hysterectomy in the United States, 1988-1990. Obstet Gynecol 83 (4): 549-555.

24. Hain JD, Linton PH, Eber HW, Chapman MM (1970) Menstrual irregularity, symptoms and personality. J Psychosom Res 14(1): 81-87.

25. Glover L, Novakovic A, Hunter M (2002) An exploration of the nature and causes of distress in women attending gynaecological outpatient clinics. J Psychosom Obstet Gynaecol 23(4): 237-248.

26. Brito LGO, Panobianco MS, Sabino-de-Freita MM, Barbosa HF, Dantas de Azevedo G, et al. (2014) Uterine leiomyoma: understanding the impact of symptoms on womens' lives. Reproductive Health 11: 10.

27. Noor S, Fawwad A, Sultana R, Bashir R, Qurat-ul-ain, et al. (2009) Pregnancy with Fibroids and Its Obstetric complications. JAyub Med CollAbbottabad 21(4): 37-40.

28. Mollica G, Pittini L, Minganti E, Perri G, Pansini F (1996) Elective uterine myomectomy in pregnant women. Clin Exp ObstetGynecol 23(3): 168172.

29. Ouyang DW, Economy KE, Norwitz ER (2006) Obstetric complication of fibroids. Obstet Gynecol Clin North Am 33(1): 153-169.

30. Vergani P, Locatelli A, Ghidini A, Andreani M, Sala F, et al. (2007) Large uterine leiomyomas and risk of cesarean delivery. Obstet Gynecol 109(2 Pt 1):410-414.

31. Nicholls C, Glover L, Pistrang N (2004) The illness experiences of women with fibroids: An exploratory qualitative study. J Psychosom Obstet Gynecol 25(3-4): 295-304.

32. Williams VS, Jones G, Mauskopf J, Spalding J, DuChane J (2006) Uterine fibroids: A review of health-related quality of life assessment. J Womens Health (Larchmt) 15(7): 818-829. 
33. Okolo S (2008) Incidence, aetiology and epidemiology of uterine fibroids. Best Practice and Research Clinical Obstetrics and Gynaecology 22(4): 571-588.

34. Peddada SD, Laughlin SK, Miner K, Guyon JP, Haneke K, et al. (2008) Growth of uterine leiomyomata among premenopausal black and white women. Proc Natl Acad Sci USA 105 (50): 19887-19892.

35. Laughlin SK, Baird DD, Savitz DA, Herring AH, Hartmann KE (2009) Prevalence of uterine leiomyomas in the first trimester of pregnancy: An ultrasound-screening study. Obstetrics and gynecology 113(3): 630635.

36. Radhika BH, Naik K, Shreelatha S, Vana (2015) Case series: Pregnancy Outcome in Patients with Uterine Fibroids. J Clin Diagn Res 9(10): QR01QR04.

37. Sarwar I, Habib S, Bibi A, Malik N, Parveen Z (2012) Clinical audit of foeto maternal outcome in pregnancies with fibroid uterus. Journal of Ayub Medical College, Abbottabad: JAMC 24(1): 79-82.
38. Stewart EA, Nowak RA (1996) Leiomyoma-related bleeding: A classic hypothesis updated for the molecular era. Hum Reprod Update 2(4): 295-306.

39. Puri K, Famuyide AO, Erwin PJ, Stewart EA, Laughlin-Tommaso SK (2014) Submucosal fibroids and the relation to heavy menstrual bleeding and anemia. Am J Obstet Gynecol 210(1): 38.e1-38.e7.

40. Bachmann GA, Bahouth LA, Amalraj P, Mhamunkar V, Hoes K, et al. (2011) Uterine fibroids: Correlations of anemia and pain to fibroid location and uterine weight. J Reprod Med 56(11-12): 463-466.

41. Ben-Nagi J, Miell J, Mavrelos D, Naftalin J, Lee C, et al. (2010) Endometrial implantation factors in women with submucous uterine fibroids. Reprod Biomed Online 21(5): 610-615.

42. Purohit P, Vigneswaran K (2016) Fibroids and Infertility. Curr Obstet Gynecol Rep 5: 81-88.

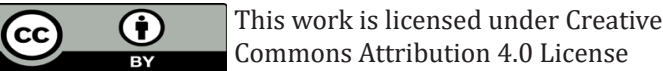

To Submit Your Article Click Here:

Submit Article
DOI: 10.32474/IGWHC.2018.02.000139

\section{Interventions in Gynecology and} Women's Healthcare

\section{IGWHC}

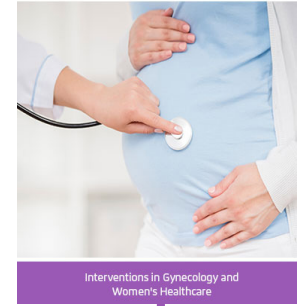

\section{Assets of Publishing with us}

- Global archiving of articles

- Immediate, unrestricted online access

- Rigorous Peer Review Process

- Authors Retain Copyrights

- Unique DOI for all articles 